\title{
BETHANECHOL CHLORIDE IN NEUROGENIC BLADDER DYSFUNCTION
}

\author{
ANANIAS C. DIOKNO, M.D. \\ RON KOPPENHOEFER, M.D.
}

From the Section of Urology, Department of Surgery, and Department of Physical Medicine and Rehabilitation, University of Michigan Medical Center,

Ann Arbor, Michigan

\begin{abstract}
Representative case summaries of patients with different types of neurogenic bladder diagnosed by combined cystometric-perineal electromyography and treated with bethanechol (Urecholine) are presented. Determination of the activity of the periurethral striated muscle in relation to bladder dysfunction is extremely important in the selection of patients for bethanechol therapy. In the absence of structural obstruction, bethanechol can be used in patients with (1) the early phase of coordinated reflex neurogenic bladder and sphincter when there is incomplete bladder emptying due to feeble or unsustained detrusor contractions, (2) recovery phase of spinal shock when the periurethral striated muscle has recovered and is under voluntary control, (3) incomplete motor paralytic bladder with coordinated sphincter, and (4) sensory paralytic bladder with decompensation. The bethanechol regimen will vary in accordance with the type of bladder being treated.
\end{abstract}

Bethanechol chloride (Urecholine)* is a parasympathomimetic agent that acts on the postganglionic parasympathetic effector cells to enhance selectively the contractions of the smooth muscle of the gastrointestinal tract and the detrusor muscle of the urinary bladder. Its clinical use has been described in postoperative urinary retention ${ }^{\mathbf{1 , 2}}$ and in chronic hypotonic dysfunction of the bladder. ${ }^{3}$

The use of bethanechol in patients with neurogenic bladder is well known. It has been used in conjunction with intermittent catheterization in patients with spinal cord injury to achieve adequate reflex voiding and a catheterfree state. It has also been used either at the onset of an intermittent catheterization program in all patients if no contraindications exist ${ }^{4,5}$ or seven to ten days later if no spontaneous voiding has occurred. ${ }^{6}$

With the advent of sophisticated instruments, accurate identification of the nature of the

*Merck, Sharp and Dohme, West Point, Pennsylvania. neurogenic bladder dysfunction is now a reality. Therefore, it is possible to screen individuals who will benefit from bethanechol and to avoid indiscriminate use of this very potent and expensive drug.

The purpose of this report is to attempt to clarify the indications for the use of bethanechol in patients with neurogenic bladder dysfunctions. Representative case summaries will be presented and their significance discussed.

\section{Case Summaries}

\section{Case 1}

A twenty-year-old white male was involved in a motorcycle accident and sustained a compression fracture of the fourth and fifth dorsal vertebrae with a resultant complete sensory and motor paraplegia of the fourth dorsal vertebra. Ten days after the injury a combined cystometric and perineal electromyographic study showed a spinal shock bladder and recovery phase of the periurethral striated muscle. ${ }^{7}$ During the combined study, attempted voiding 
produced no detrusor contractions or relaxation of the sphincter.

The paticnt was placed on an intermittent self-catheterization program. ${ }^{8}$ One month later a follow-up cystometric-electromyographic study showed a reflex bladder with coordinated detrusor and sphincter. ${ }^{7}$ During the study, it was observed that the detrusor contractions were too weak and transient to empty the bladder completely in spite of complete relaxation of the periurethral striated muscle. Endoscopy revealed no obstruction. He was then taught various methods to trigger reflex voiding. In addition, he was placed on $25 \mathrm{mg}$. of bethanechol orally every six hours and instructed to catheterize himself mainly for check of residual urine. He did extremely well on this program; his urine remained uninfected and residual urines were low. Bethanechol was discontinued without difficulty eight months after injury.

\section{Case 2}

Complete paraplegia of the fourth dorsal vertebra and acute urinary retention suddenly developed in a thirty-year-old male. He was transferred to the University of Michigan Medical Center and a laminectomy (third to fourth dorsal vertebra) was performed; the pathology report was consistent with multiple myeloma. $\mathrm{He}$ received postoperative radiation and chemotherapy but his paraplegia remained unchanged.

A cystometric-electromyographic study one month after the laminectomy showed a reflex neurogenic bladder with an uncoordinated sphincter. Initial therapy consisted of intermittent self-catheterization and an anticholinergic agent. After more than two years of selfcatheterization without any problem, urinary incontinence developed between catheterizations. A repeat combined study showed a reflex neurogenic bladder with coordinated sphincter activity. Reflex voiding was augmented with bethanechol $25 \mathrm{mg}$. orally four times daily. Residual urines were approximately 50 to $75 \mathrm{ml}$. after the sixth day of this regimen. Bethanechol was discontinued a month later when follow-up visits showed low residual urines.

\section{Case 3}

A thirty-year-old female achondroplastic dwarf was admitted with spastic paraparesis of the eighth dorsal vertebra secondary to dorsolumbar stenosis. A decompressive laminectomy from eighth dorsal to third lumbar vertebra was performed but paraparesis remained.
A cystometric-electromyographic study two weeks later indicated upper and lower motor neuron involvement and a positive bethanechol test. Electromyography of the periurethral striated muscle revealed a spastic sphincter with incomplete relaxation during attempted micturition. She was initially treated by intermittent self-catheterization and an anticholinergic agent. Howcver, bccause of her stature catheterization was difficult and had to be terminated. Bethanechol, $5 \mathrm{mg}$. given subcutaneously, was associated with severe side effects; the dosage was then adjusted to $2.5 \mathrm{mg}$. subcutancously every four hours. She voided small volumes ranging from 25 to $30 \mathrm{ml}$. and had residual urines of 250 to $300 \mathrm{ml}$. Bethanechol therapy was discontinued, and the patient was placed again on an indwelling Foley catheter.

\section{Case 4}

A twenty-six-year-old black male was shot in the back. Physical examination showed anesthesia and analgesia on the right from the fourth lumbar to first sacral vertebra and anesthesia of the first sacral vertebra on the left. Motor examination revealed paralysis of the right lower extremity from the fourth lumbar vertebra. Combined cystometry and electromyography showed an incomplete motor paralytic bladder with partial denervation of the periurethral striated muscle. During this combined study, it was demonstrated that attempted voiding produced no voiding contractions but resulted in relaxation of the periurethral striated muscle. Bethanechol $(7.5 \mathrm{mg}$. subcutaneously every six hours) was given, and the patient voided spontaneously with residual urines in the 30 - to 100 - $\mathrm{ml}$. range. Bethanechol was reduced to 5 mg. subcutaneously every six hours three days later. The residual urine remained low on this dosage, and after two days bethanechol was changed to $50 \mathrm{mg}$. orally every six hours. The patient continued to void adequately with low residuals of 35 to $40 \mathrm{ml}$., and the bethanechol was discontinued after several more days.

\section{Case 5}

A nineteen-year-old white male suffered a flexion injury to his cervical spine with a resultant dislocation at the fourth to fifth cervical vertebra. He underwent a laminectomy on May 6, 1975. On June 3, 1975, the patient was transferred to the University of Michigan Medical Center, and physical examination at that time showed fair to good muscle strength in all 
extremities, clonus in lower extremities, and bilateral Hoffmann and Babinski signs. Cystometrograms and electromyograms showed a spinal shock bladder with recovery phase of the striated musculature. The periurethral striated muscle showed increasing electrical activity with bladder distention. Attempts at voiding produced voluntary relaxation of the striated muscle without any noticeable change in the intravesical pressure. He was treated with bethanechol $2.5 \mathrm{mg}$. subcutaneously every four hours. With this dosage abdominal cramps developed, and the dose was then reduced to 25 mg. orally every six hours. He urinated with high residual urines, and after a week the bethanechol dosage was increased to $50 \mathrm{mg}$. orally every six hours. He responded well to this dose and voided with volumes of $300 \mathrm{ml}$. and residual urines of 40 to $50 \mathrm{ml}$. Bethanechol was discontinued one month later; residual urines were 50 to $70 \mathrm{ml}$., and voided volumes were 300 to $350 \mathrm{ml}$.

\section{Comment}

Normal micturition is a complex process which requires integration of a central neuronal network with a peripheral neuromuscular system. The end result is a voluntary coordinated detrusor contraction and periurethral striated muscle relaxation. The central neuronal network, responsible for the volitional control, consists of pathways between the white and gray matter of the frontal lobes, specific thalamic nuclei, basal ganglia, and the cerebellum to the second to fourth sacral segments. The peripheral neuromuscular component consists of a reflex arc involving the second to fourth sacral segments with motor axons to the detrusor passing via the pelvic nerve, and motor axons to the periurethral striated musculature passing via the pudendal nerve.

A lesion of any of the neurologic components of this reflex may result in vesical dysfunction, a condition which is called neurogenic bladder. ${ }^{9}$ After the initial insult to the spinal cord, a majority of the patients go through a period of spinal shock, when all neural reflexes below the level of the injury are absent. The return of reflex activity varies in both time and type; striated muscle activity usually recovers earlier than smooth muscle activity. ${ }^{7}$

Spinal shock can be divided into two phases, acute and recovery. During the acute phase the bladder exhibits no contractions and the periurethral striated muscle no electrical activity.
During this acute phase, bethanechol is ineffective. In the recovery phase of spinal shock, the bladder is still inactive, but now the periurethral striated muscle will reveal varying degrees of electrical activity.

In incomplete lesions of the spinal cord above the sacral reflex arc, voluntary relaxation of the periurethral striated muscle can occur without detrusor contraction and may be elicited by asking the patient to attempt voiding. This indicates that voluntary somatic control to the sphincter has recovered or is in the recovery phase. We have found bethanechol extremely helpful in rehabilitating this type of bladder (Case 5). The dose starts with 2.5 to $5 \mathrm{mg}$. of bethanechol subcutaneously every four to six hours with catheterization for residuals once or twice a day. When residual urine is less than 50 $\mathrm{ml}$., the dose is converted to either $2.5 \mathrm{mg}$. subcutaneously or directly to $50 \mathrm{mg}$. orally every six hours. After a minimum period of one week, the dose is tapered and ultimately discontinued if residual urine remains low. Not infrequently it may be necessary to administer the oral bethanechol for a number of weeks or months before satisfactory voiding is attained and the drug can be discontinued.

In patients with complete spinal cord lesion above the second to fourth sacral segments, an involuntary micturition reflex will appear weeks or months after the injury. The combined cystometric-electromyographic study has shown two types of reflex neurogenic bladders, coordinated and uncoordinated detrusor and sphincter. The uncoordinated type is obviously not suited for bethanechol and reflex voiding because of the functional obstruction created by the contraction of the striated musculature during detrusor contraction (Case 3). The bladder with coordinated detrusor and sphincter is ideal for reflex voiding. We have used bethanechol in the early phase of the trial voiding if during the combined study the detrusor contractions are weak (low amplitude) or not sustained (Cases 1 and 2), leading to incomplete bladder emptying. Endoscopy should be performed to rule out outlet obstruction.

Because paraplegic patients are supersensitive to bethanechol, smaller oral doses are usually effective in producing stronger detrusor contractions. An initial dose of $25 \mathrm{mg}$. orally every six hours is used, and the dosage is increased or reduced depending on the response of the individual. Once reflex voiding is fully established the drug is withdrawn, and the patient 
is examined periodically to be certain that frequent complete bladder emptying is maintained.

Injuries involving sacral segments two to four or the pelvic nerve may produce either a complete or incomplete lower motor neuron bladder. The patients with complete motor paralytic bladder and autonomous neurogenic bladder are not suitable for bethanechol therapy because they are either totally incontinent from absence of sphincter control or lack the necessary coordination between the detrusor and sphincter. Patients with incomplete lesions may be candidates for bethanechol therapy if the partial motor paralytic bladder is associated with relaxation of the periurethral striated muscle during attempts at voiding. In this situation incomplete bladder emptying can be improved by bethanechol (Case 4). In cases of absent or very weak voluntary detrusor contractions, we have utilized higher doses of bethanechol as recommended by Lapides ${ }^{10}$ to rehabilitate the bladder. An inital dose of 7.5 to $10 \mathrm{mg}$. subcutaneously every four to six hours in adults was found necessary to effect adequate emptying. The dose is then tapered every three days, $2.5 \mathrm{mg}$. at a time, to a minimum dose of $5 \mathrm{mg}$. subcutaneously every four to six hours. After three days and residual urines of less than $50 \mathrm{ml}$. the dosage is changed to $50 \mathrm{mg}$. orally every six hours. The patients are maintained on this dosage if attempts to reduce it are unsuccessful.

The decompensated bladders observed in patients with sensory paralytic bladder are managed in the same manner as the incomplete motor paralytic bladders. In this condition the problem is the inability of the detrusor to produce effective contraction to empty the bladder despite the periurethral striated muscle relaxing during the attempted voiding. The success of bethanechol therapy will depend on the extent of detrusor decompensation.

Ann Arbor, Michigan 48104

(DR. DIOKNO)

\section{References}

1. Starr, I., and Ferguson, L. K.: Methylcholine urethane: its action in various normal and abnormal conditions, especially postoperative urinary retention, Am. J. Med. Sci. 200: 372 (1940).

2. Garvey, F. D., Bowman, M. D., and Alsobrook, W. L.: The use of beta-methylcholine urethane in postoperative urinary retention, Surg. Gynecol. Obstet. 88: 96200 (1949).

3. LEE, L. W.: The clinical use of urecholine in dysfunctions of the bladder, J. Urol. 62: 300 (1949).

4. Bors, E.: Intermittent catheterization in paraplegic patients, Urol. Int. 22: 236 (1968).

5. Firlit, C. F., et al.: Experience with intermittent catheterization in chronic spinal cord injury patients, J. Urol. 114: 234 (1975).

6. Perkash, I.: Intermittent catheterization and bladder rehabilitation in spinal cord injury patients, ibid. 114: 230 (1975).

7. Diokno, A. C., Koff, S. A., and Bender, L.: Periurethral striated muscle activity in neurogenic bladder dysfunction, ibid. 112: 743 (1974).

8. Lapides, J., Diokno, A. C., Lowe, B. S., and KaLISH, M.: Follow-up on unsterile, intermittent self-catheterization, ibid. 111: 184 (1974).

9. LAPIDES, J.: Neuromuscular vesical and ureteral dysfunction, in Campbell, M. F., and Harrison, J. H., Eds.: Urology, 3rd ed., Philadelphia, W. B. Saunders Company, 1970, chap. 33, pp. 1343-1378.

10. IDEM: Urecholine regimen for rehabilitating the atonic bladder, J. Urol. 91: 658 (1964). 